\title{
An improved nerve-sparing radical hysterectomy technique for cervical cancer using the paravesico-vaginal space as a new surgical landmark
}

\author{
Yuqin Zhang ${ }^{1, *}$, Tingyan Shi ${ }^{1, *}$, Sheng Yin ${ }^{1}$, Sining Ma ${ }^{1}$, Di Shi ${ }^{1}$, Jun Guan ${ }^{2,3}$, Libing \\ Xiang ${ }^{4}$, Yang Liu ${ }^{4}$, Yulan Ren ${ }^{4}$, Deyan Tan ${ }^{5}$ and Rongyu Zang ${ }^{1}$ \\ ${ }^{1}$ Division of Gynecologic Oncology, Department of Obstetrics and Gynecology, Zhongshan Hospital, Fudan University, \\ Shanghai, China \\ ${ }^{2}$ Department of Gynecology, Tumor Bank Ovarian Cancer, European Competence Center for Ovarian Cancer, Campus Virchow \\ Clinic, Charité Medical University of Berlin, Berlin, Germany \\ ${ }^{3}$ Nuffield Department of Obstetrics and Gynecology, University of Oxford, Oxford, United Kingdom \\ ${ }^{4}$ Department of Gynecologic Oncology, Fudan University Cancer Center, Shanghai, China \\ ${ }^{5}$ Department of Anatomy, Shanghai Medical College, Fudan University, Shanghai, China \\ *These authors contributed equally to the work \\ Correspondence to: Rongyu Zang, email: ryzang@yahoo.com
}

Keywords: paravesico-vaginal space, nerve-sparing radical hysterectomy, deep uterine vein, terminal ureter, cervical cancer

Received: April 11, 2017 Accepted: June 16, $2017 \quad$ Published: July 05, 2017

Copyright: Zhang et al. This is an open-access article distributed under the terms of the Creative Commons Attribution License 3.0 (CC BY

3.0), which permits unrestricted use, distribution, and reproduction in any medium, provided the original author and source are credited.

\section{ABSTRACT}

Bladder dysfunction remains a major postoperative challenge for early stage cervical cancer patients. The present prospective phase 2 trial in patients with stage IB1 and IIA1 cervical cancer follows up on our previous, unpublished work describing a new surgical landmark, the paravesico-vaginal space. We describe a novel nervesparing radical hysterectomy (NSRH) approach to treat early stage cervical cancer without compromising local control rate or survival. Between September 2015 and August 2016, 49 patients were enrolled to receive NSRH. The bladder catheter was routinely removed on postoperative day 4 . The primary endpoints were rate of postvoid residual urine volume (PVR) $\leq 50 \mathrm{ml}$ and proportion of patients with successful catheter removal (ClinicalTrials.gov Identifier: NCT02562729). Anatomically, from ventral to dorsal, the terminal ureter, deep uterine vein, and cardinal ligament were the three markers of the paravesico-vaginal space. The median operative time was $100 \mathrm{~min}$, and the median blood loss was $200 \mathrm{ml}$. Thirty-four patients $(69.4 \%)$ had successful catheter removal on postoperative day 4 , and 17 patients $(34.7 \%)$ had a PVR $\leq 50 \mathrm{ml}$. Our results suggest that by accessing the paravesico-vaginal space landmark, the bladder branch of the inferior hypogastric plexus can be completely preserved, contributing to greater NSRH efficiency without compromising outcomes for patients with early stage cervical cancer.

\section{INTRODUCTION}

The first radical hysterectomy with lymphadenectomy for cervical cancer was performed by Wertheim in Vienna in $1898[1,2]$. Over the next five decades, Okabayashi and Meigs improved the safety and radicality of this approach, which became the foundation of radical hysterectomy for invasive cervical cancer [3-5]. Ever since, however, only few reports described detailed procedures for complete resection of the parametrium $[6,7]$.

Due to the extent of parametrial or cardinal ligament transection, the classical surgical management is usually followed by urinary dysfunction because of the injury caused to the pelvic autonomic nerves [8-10]. This is of 
great concern, inasmuch as preservation of autonomic nerve fibers during radical hysterectomy minimizes postoperative bladder morbidity and improves the quality of life in cervical cancer patients [11-14]. In 2006, we firstly reported an evidence-based nerve-sparing radical hysterectomy (NSRH) technique in China [15, 16], and then improved this approach in our clinical practice. Between 2011 and 2014, we conducted a preliminary retrospective study to evaluate its efficiency and safety (unpublished data).

In 2007, Fujii et al. reported their NSRH procedures in detail by describing the precise anatomy of the pelvic nerves under magnification [17, 18]. In 2008, Querleu and Morrow defined NSRH as type C1 in their new classification system for radical hysterectomy [19]. However, due to the complexity of the pelvic anatomy, no global uniformity was reached about NSRH surgical procedures, particularly in regards to preservation of the bladder branch of the inferior hypogastric plexus (IHP).

Through practicing on fresh cadavers and patients who desired to preserve their bladder function, we made a breakthrough finding represented by a novel clinical anatomic landmark, the paravesico-vaginal space. By incorporating this surgical landmark into radical hysterectomy, we simplified nerve-sparing procedures by achieving: i) complete preservation of the IHP without dissecting any nerve plexus; ii) precision of parametrial transection; and iii) a shorter operation time. In our earlier exploratory study using this surgical approach, we registered successful catheter removal in $75.0 \%$ and in $85.2 \%$ of patients on postoperative day 4 and day 7 , respectively (unpublished data). Following these encouraging surgical and clinical outcomes, the present prospective study was carried out between 2015 and 2016 to further validate the use of the paravesico-vaginal space landmark to improve the efficiency of NSRH.

\section{MATERIALS AND METHODS}

\section{Study design and participants}

This prospective, phase 2 trial was conducted in the Zhongshan Hospital, Fudan University (FDUZH). Each patient provided written informed consent before participation. This study was approved by the Ethics Committee of FDUZH, and registered with ClinicalTrials. gov (NCT02562729).

Between September 6, 2015 and August 23, 2016, patients were enrolled if they met the following inclusion criteria: FIGO stage IB1 and IIA1 cervical cancer; age between 18 and 70 years; histologically confirmed primary adenocarcinoma, squamous cell carcinoma, or adenosquamous carcinoma of the uterine cervix; no neoadjuvant chemotherapy; Karnofsky performance status $\geq 70$; compliance with follow-up; provision of written informed consent. The exclusion criteria were as follow: age $<18$ or $>70$ years; history of lower urinary tract damage or surgery; abnormal urodynamic study results; Karnofsky performance status $<70$; patients with uncontrolled psychological disorders; patients unwilling or unable to comply with the protocol; prior treatment with pelvic radiotherapy. All patients were preoperatively evaluated by pelvic magnetic resonance imaging (MRI) and abdominal computed tomography (CT).

All enrolled patients received NSRH with catheter removal routinely performed on postoperative day 4 . Postvoid residual urine volume (PVR) was measured by ultrasound after spontaneous voiding. The standards for recatheterization were as follow: i) patients unable to void spontaneously or showing voiding difficulty; or ii) patients with PVR > $200 \mathrm{ml}$. In these cases, the reset catheter was removed again on postoperative day 14 . Patients who did not need recatheterization received subsequent testing by ultrasound until the PVR was $<100 \mathrm{ml}$ or until satisfaction of micturition or sensation of bladder fullness were achieved.

\section{Procedures}

NSRH or nerve-sparing radical trachelectomy was followed by pelvic lymphadenectomy. Crucial surgical procedures necessary to identify the paravesico-vaginal space landmark are shown in Supplementary Table 1.

Adjuvant concurrent chemoradiotherapy (CCRT) or CCRT followed by chemotherapy using cisplatin and paclitaxel was administered according to the following criteria: more than two intermediate-risk factors, including i) pathological tumor size $\geq 4 \mathrm{~cm}$, ii) stromal invasion $\geq 1 / 2$, and iii) lymphovascular space invasion (LVSI); or more than one high-risk factor, including i) lymph node metastasis, ii) parametrial invasion, and iii) positive resection margin.

\section{Outcomes}

The primary endpoints of this study were rate of $\mathrm{PVR} \leq 50 \mathrm{ml}$ and proportion of patients in which the catheter was removed successfully on postoperative day 4 .

\section{Statistical analysis}

Sample size considerations: The unpublished results from our previous retrospective study showed that the proportion of patients with a PVR $\leq 50 \mathrm{ml}$ on postoperative day 4 increased from $10 \%$ to $39.8 \%$ after receiving our modified NSRH technique incorporating the paravesicovaginal space landmark. Considering a type I error rate of 0.05 and a power of $90 \%$, we planned to recruit 43 patients in the current prospective phase 2 study, a sample size calculated using the PASS software (version 11.0).

Medical records were abstracted for age, body mass index (BMI), FIGO stage, histology, operative time, estimated blood loss, postoperative hospital stay, 
pathology, postoperative 30-day morbidity, duration of postoperative catheterization (DPC), and PVR. BMI values were classified according to the WHO criteria [21]. Statistical computing was performed with SPSS software package for Windows (Statistical Package for the Social Sciences 19.0, SPSS Inc, Chicago, IL).

\section{Roles of the funding source}

The funding agency supporting this study had no involvement in study design, patient recruitment, data collection, data analysis, data interpretation, or writing of the report. The corresponding author had full access to all data in the study and all authors share responsibility for the decision to submit it for publication.

\section{RESULTS}

\section{Baseline and patient characteristics}

Between September 6, 2015, and August 23, 2016, 49 patients were enrolled in this trial and all of them underwent NSRH and pelvic lymphadenectomy. Table 1 summarizes the clinical and pathological characteristics of the patients. The median age was 53 years (range: 34-70 years). Four patients were categorized as underweight with BMI $<18.5$; 31 were normal weight or at risk of overweight with BMI 18.5-24.9; ten patients were obese class I with BMI 25-29.9; and four patients were obese class II with BMI $\geq 30$ [21]. Twenty-six patients $(53.1 \%)$ were diagnosed with stage Ib1, and 23 (46.9\%) with stage IIa1 cervical cancer. Histology revealed 47 cases $(95.9 \%)$ of squamous cell carcinoma, one case of adenocarcinoma, and one case of adenosquamous carcinoma.

\section{Surgical anatomy of the paravesico-vaginal space}

After resecting the uterine artery, we dissected the ureteral tunnel, a free space covered by the ventral portion of the vesicouterine ligament. Then, we accessed the first layer's marker, namely the terminal ureter at the apex of the paravesico-vaginal space. On the second layer, the site of both the bladder and uterine branches of the IHP, we cut the dorsal portion of the vesicouterine ligament together with the deep uterine vein, which was the second marker. A precision sharp dissection was performed to protect all bladder branches of the IHP by pushing them laterally and caudally. Finally, the cardinal ligament was accessed at the bottom border of the paravesico-vaginal space, which was the third marker (Table 2, Figure 1, Supplementary Figure 1, and Supplementary Table 1).

\section{Surgical outcomes}

As shown in Table 1, the median operative time was $100 \mathrm{~min}$, ranging from 40 to $175 \mathrm{~min}$. The median estimated blood loss was $200 \mathrm{ml}$ (range: 100-2200 ml). The median postoperative hospital stay was 7 days, ranging from 5 to 13 days. Five patients $(10.2 \%)$ presented a pathological tumor diameter $>4 \mathrm{~cm}$. Twenty-four (49.0\%) and 29 (59.2\%) patients presented positive LVSI and deep stromal invasion, respectively. Ten patients $(20.4 \%)$ presented lymph node metastasis, and one patient $(2.0 \%)$ showed suspicious positive resection margin.

Primary outcomes of this study are shown in Table 3. Among the 49 patients, 34 (69.4\%) had successful catheter removal on postoperative day 4 , and $15(30.6 \%)$ received recatheterization. Seventeen of 34 patients $(50.0 \%)$ who underwent successful catheter removal showed a PVR $\leq 50 \mathrm{ml}$. Although three patients showed a PVR $>200 \mathrm{ml}$, all of them reported sensation of bladder filling and satisfaction of micturition. Among the 21 patients with a PVR of 50-200 ml, 14 (66.7\%) had successful catheter removal on postoperative day 4 and were followed up daily by ultrasound. Thirteen patients had a PVR $<100 \mathrm{ml}$ three days later, and the other one who rejected recatheterization had a PVR $<100 \mathrm{ml}$ on postoperative day 14. Among the 15 patients who received recatheterization, the DPC was 14 days in 13 patients, and 20 and 21 days, respectively, in the other two.

In total, 13 patients $(26.5 \%)$ had postoperative complications according to the Common Terminology Criteria for Adverse Events (CTCAE) v4.03, but none reported with grade III-V morbidity or mortality according to the Memorial Sloan-Kettering Cancer Center (MSKCC) surgical secondary events grading system. As shown in Table 4, the most common complication was hemorrhage ( $n=3,6.1 \%)$, with one patient suffering an intraoperative hemorrhage with an estimated blood loss of $2200 \mathrm{ml}$. Two patients had postoperative hemorrhage according to regular hemoglobin test on peripheral blood and the volume of drainage. Other morbidities included: 2 cases of pelvic infections, 3 of urinary infections, 2 of incision infections, 1 of venous thrombosis, 1 of urinary incontinence, 1 of lymphocyst, and 1 case of lymphorrhagia. One patient suffered from both postoperative hemorrhage and pelvic infection.

\section{DISCUSSION}

\section{Dissection of the ventral parametrium}

Since its introduction more than 70 years ago by Meigs and Okabayashi, resection of the parametrium via the paravesical space became a widely adopted technique among gynecologists worldwide. Although commonly reported in the literature, this approach was infrequently utilized during NSRH procedures because the bladder branch of the IHP is always partly damaged by either laparotomy or laparoscopic surgery. In Fujii's study, after the development of the paravesical and pararectal space, the uterine artery and the superficial uterine vein were isolated and transected close to the pelvic sidewall 
Table 1: Clinicopathological characteristics $(N=49)$

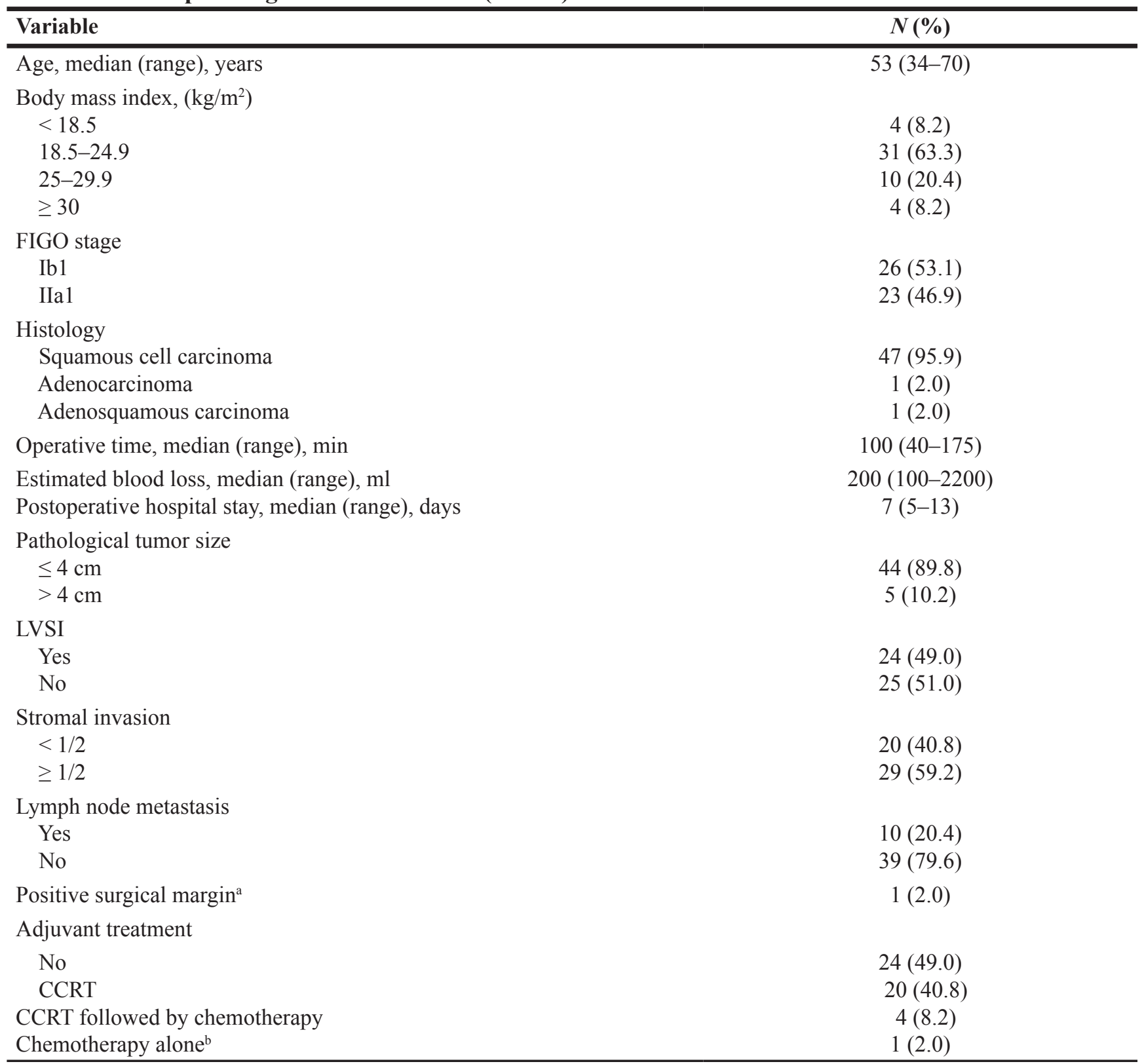

Abbreviations: FIGO, International Federation of Gynecology and Obstetrics; LVSI, lymph-vascular space invasion; CCRT, concurrent chemoradiation

${ }^{a}$ One case showed suspicious positive vaginal surgical margin according to pathological findings.

bone patient with LVSI underwent abdominal trachelectomy.

$[17,18]$. In another report, the paravaginal space was developed to find the dorsal vesicouterine ligament running between the paravaginal and paravesical spaces, and was mainly utilized for separating and transecting the middle and inferior vesical veins [22].

Notably, although Wertheim described in 1898 the resection of the parametrium via the medial ureter without dissecting the lateral components [1], no surgeon, to our knowledge, reported using his procedures thereafter. The current prospective study is thus the first to demonstrate that it is feasible to resect enough length of the parametrium via the inner of the terminal ureter by dividing an avascular landmark, the paravesico-vaginal space, a crucial procedure for total preservation of the bladder branch of the IHP.

\section{Development of the paravesico-vaginal space and its anatomic boundaries}

Querleu and Morrow classified the nerve-sparing modality of radical hysterectomy as type $\mathrm{C} 1$ [19], but the bladder branch of the IHP was still injured during these 
Table 2: Anatomic hierarchy of the paravesico-vaginal space, from ventral and caudal to dorsal and cranial

\begin{tabular}{ll}
\hline Hierarchical Anatomical markers & \\
\hline LEVEL ONE & ventral portion of the vesicouterine ligament \\
& uterine artery, ureteral tunnel \\
& terminal ureter (first anatomic marker) \\
LEVEL TWO & dorsal vesicouterine ligament \\
& deep uterine vein (second marker) \\
& inferior hypogastric nerve plexus \\
LEVEL THREE & paravesico-vaginal space, cardinal ligament (third marker) \\
& inferior vesical vein \\
\hline
\end{tabular}

procedures due to the uncertain relationship between the ventral parametrium and the IHP. Over the past decade, we explored and developed a more concise approach to preserve the IHP, and defined a new anatomic landmark for dissecting the ventral and medial parametrium using the modified Wertheim's method.

In contrast with most published techniques, where the deep uterine vein is used as the landmark for identification and preservation of the pelvic splanchnic nerves, the novel landmark proposed here comprises an avascular space. The paravesico-vaginal space was developed quickly and with extreme ease from the terminal ureter, after which we
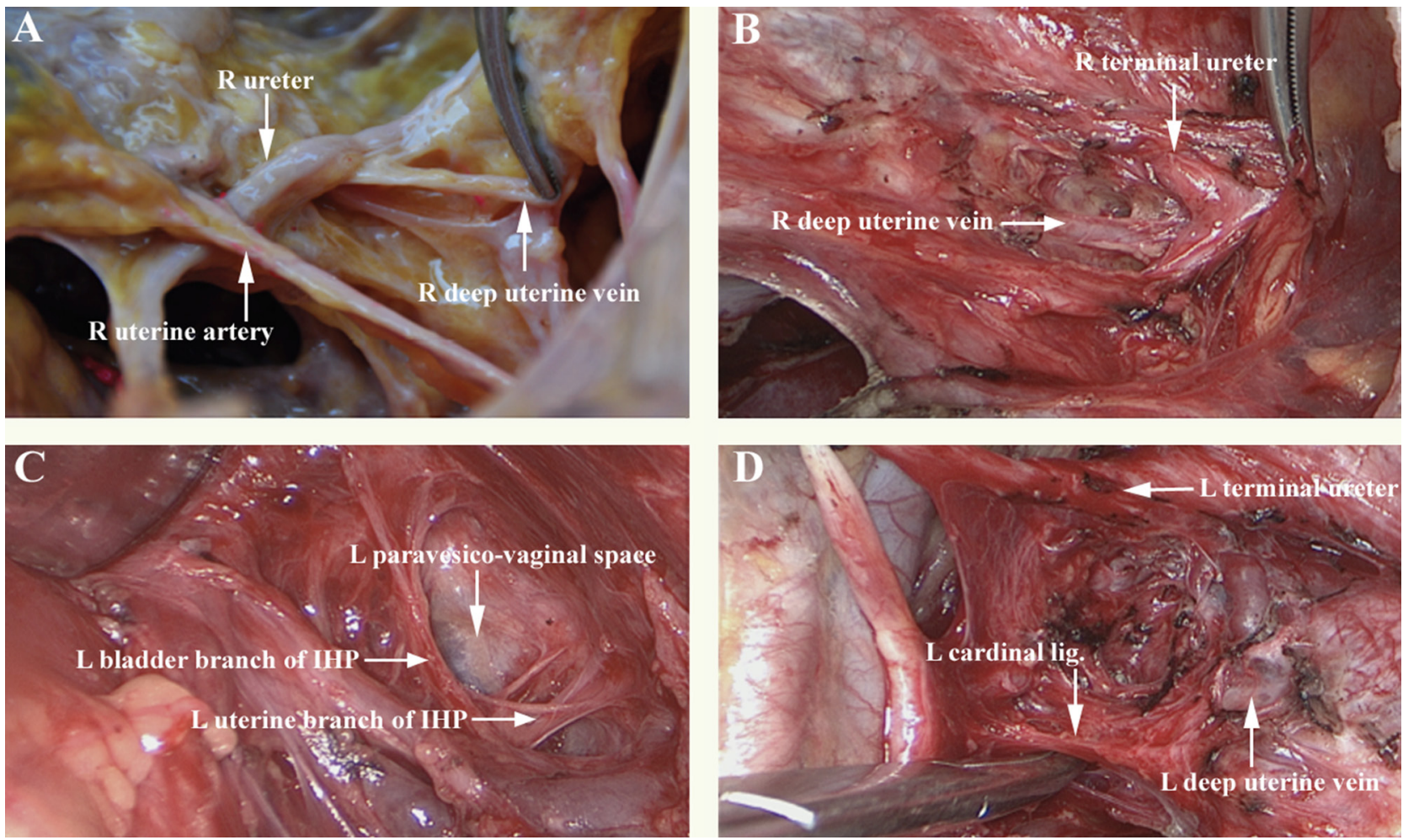

Figure 1: Anatomic relationship between the paravesico-vaginal space and the deep uterine vein. (A) right, anatomical view of fresh cadavers; (B) right, ventral and cranial operative view (level one); (C) left lateral, ventral, and cranial operative view (level two); (D) left, operative view of the pear-shaped paravesico-vaginal space and the cardinal ligament (level three). Abbreviations: IHP, inferior hypogastric plexus; R, right; L, left. 
Table 3: Status of bladder function recovery

\begin{tabular}{lc}
\hline Variable & N(\%) \\
\hline Catheter removal on POD 4 & $34(69.4)$ \\
Success & $15(30.6)$ \\
Failure & $17(34.7)$ \\
PVR $\leq 50 \mathrm{ml}$ on POD 4 & $32(65.3)$ \\
$\quad$ Yes & $17(50.0)$ \\
No & $14(41.2)$ \\
Successful catheter removal on POD 4 & $3(8.8)^{\mathrm{a}}$ \\
PVR $\leq 50 \mathrm{ml}$ & \\
$50 \mathrm{ml}<$ PVR $\leq 200 \mathrm{ml}$ & $13(86.7)$ \\
PVR $>200 \mathrm{ml}$ & $2(13.3)$ \\
Catheter removal on POD 14 & \\
Success & \\
Failure & \\
\hline
\end{tabular}

Abbreviations: POD, Postoperative Day; PVR, postvoid residual urine volume.

${ }^{a}$ All the 3 patients had sensation of bladder filling and satisfaction of micturition. The PVR was less than $100 \mathrm{ml}$ on POD 7 in two patients and on POD 8 in one patient.

Table 4: Assessment of complications 30 days after surgery

\begin{tabular}{lcc}
\hline Complication & $\mathbf{N}$ & $\mathbf{\%}$ \\
\hline Hemorrhage & 3 & 6.1 \\
$\quad$ Intraoperative hemorrhage & 1 & \\
$\quad$ Postoperative hemorrhage & 2 & 4.1 \\
Pelvic infection & 2 & 6.1 \\
Urinary tract infection & 3 & 4.1 \\
Wound infection & 2 & 2.0 \\
Deep venous thrombosis & 1 & 2.0 \\
Urinary incontinence & 1 & 2.0 \\
Lymphocele & 1 & 2.0 \\
Lymphorrhagia & 1 & 0 \\
MSKCC Grade III/IV & 1 & 0 \\
Mortality (MSKCC Grade V) & 0 & 26.5 \\
Total & 0 & $13^{\mathrm{c}}$ \\
\hline
\end{tabular}

a'Both patients were diagnosed with postoperative hemorrhage because of the following reasons: i) presence of bloody, continuous peritoneal drainage; ii) hemoglobin value decreased by $10 \mathrm{~g} / \mathrm{L}$ in 24 hours on postoperative days 1-2.

${ }^{b}$ Memorial Sloan-Kettering Cancer Center surgical secondary events grading system.

'One case presented two complications.

portion, the cardinal ligament at its bottom border, a bridge connecting with the deep uterine vein, and the IHP laterally.

\section{Comparison with previous NSRH procedures}

Most previous NSRH reports include the detailed surgical procedures described by Fujii $[17,18]$, that clearly identify all the pelvic autonomic nerve fibers.
During these procedures, vascular damage and bleeding was always inevitable, greatly increasing the difficulty to identify and preserve the nerves. The paravesico-vaginal space is an avascular, loose space amid blood vessels, nerves, and connective tissues. After incorporating this landmark, NSRH was simplified and, compared with the conventional technique, higher efficiency was achieved in terms of both surgical time and proportion of patients with $\mathrm{PVR} \leq 50 \mathrm{ml}$ on postoperative day 4 . The main differences 
between the conventional and this novel NSRH are summarized in Supplementary Table 2.

\section{Efficiency and safety of NSRH}

To validate the efficiency of our novel NSRH technique for early stage cervical cancer, we designed this prospective study evaluating the rate of catheter removal and PVR $\leq 50 \mathrm{ml}$ on postoperative day 4 as primary end points. Successful catheter removal was accomplished in $69.4 \%$ of patients, while $34.7 \%$ showed a PVR $\leq 50 \mathrm{ml}$ (Table 3). Thus, the functional efficiency of NSRH in this study was superior than that of most previous reports in the literature $[13,16,17,20,22-25]$. For example, in Charoenkwan's study, 21 patients received NSRH with systematic pelvic lymphadenectomy, and only 6 patients $(27 \%)$ had a PVR $\leq 50 \mathrm{ml}$ upon removal of the catheter on postoperative day 7 [26].

Our median operation time was $100 \mathrm{~min}$. What's more, the median estimated blood loss was $200 \mathrm{ml}$. We find these results encouraging when compared to previous studies using sophisticated devices, such as the cavitron ultrasonic surgical aspirator (CUSA), laparoscopic neuronavigation (LANN), or other techniques [22, 23, 25]. Whereas only one patient had intraoperative complications in our study, 13 (26.5\%) showed some kind of morbidity by postoperative day 30 . This record emphasizes the need for careful postoperative management in these patients.

In conclusion, our study introduces a novel anatomical landmark, the paravesico-vaginal space, that improves the efficacy of nerve-sparing hysterectomy. We believe our approach will help simplify conventional radical hysterectomy and decrease postoperative mortality and morbidity in patients with early stage cervical cancer.

\section{Authors' contributions}

Conception and design: RYZ, DYT; Administrative support: RYZ, YQZ; Collection and assembly of data: SY, SNM, LBX, YL, YQZ, TYS; Data analysis and interpretation: RYZ, YQZ, DYT, SY, SNM, JG, TYS; Manuscript writing and revising: RYZ, YQZ, SY, SNM, JG, DS, TYS; Final approval of manuscript: All authors.

\section{ACKNOWLEDGMENTS}

This trial is registered with ClinicalTrials.gov, number NCT02562729, and was supported by funding from the Zhongshan Development Program (Grant No. 016). We would like to thank Dr. Kenneth Hatch from Arizona University for his valuable comments on this manuscript.

\section{CONFLICTS OF INTEREST}

The authors declare no conflicts of interest.

\section{REFERENCES}

1. Wertheim E. A discussion on the diagnosis and treatment of cancer of the uterus. Brit Med J. 1905; 2:689-704.

2. Wertheim E. The extended abdominal operation for carcinoma uteri (based on 500 operative cases). Am J Obstet Dis Women Child. 1912; 66:169-232.

3. Okabayashi H. Radical abdominal hysterectomy for cancer of the cervix uteri. Surg Gynecol Obstet. 1921; 33:335-41.

4. Meigs JV. Carcinoma of the cervix - the Wertheim operation. Surg Gynecol Obstet. 1944; 78:195-98.

5. Piver MS, Rutledge F, Smith JP. Five classes of extended hysterectomy for women with cervical cancer. Obstet Gynecol. 1974; 44:265-72.

6. Yabuki Y, Asamoto A, Hoshiba T, Nishimoto H, Kitamura S. Dissection of the cardinal ligament in radical hysterectomy for cervical cancer with emphasis on the lateral ligament. Am J obstet Gynecol. 1991; 164:7-14.

7. Yabuki Y, Asamoto A, Hoshiba T, Nishimoto H, Nishikawa Y, Nakajima T. Radical Hysterectomy: An Anatomic Evaluation of Parametrial Dissection. Gynecol Oncol. 2000; $77: 155-63$.

8. Forney JP. The effect of radical hysterectomy on bladder physiology. Am J Obstet Gynecol. 1980; 138:374-82.

9. Asmussen M, Heintz APM, Criffiths CT, Trimbos JB. Urodynamics after radical surgery for carcinoma of uterine cervix. Surgery in Gynecol Oncol. 1984; 16:143-164.

10. Wit EM, Horenblas S. Urological complications after treatment of cervical cancer. Nat Rev Urol. 2014; 11:110-7.

11. Kobayashi T. Abdominal radical hysterectomy with pelvic lymphadenectomy for cancer of the cervix. Tokyo: Nanzando. 1961; 178-87.

12. Kato T, Murakami G, Yabuki Y. A new perspective on nerve-sparing radical hysterectomy: nerve topography and over-preservation of the cardinal ligament. Jpn J Clin Oncol. 2003; 33:589-91.

13. Rob L, Halaska M, Robova H. Nerve-sparing and individually tailored surgery for cervical cancer. Lancet Oncol. 2010; 11:292-301.

14. Kim HS, Kim TH, Suh DH, Kim SY, Kim MA, Jeong CW, Hong KS, Song YS. Success Factors of Laparoscopic Nerve-sparing Radical Hysterectomy for Preserving Bladder Function in Patients with Cervical Cancer: A Protocol-Based Prospective Cohort Study. Ann Surg Oncol. 2015; 22:1987-1995.

15. Zang RY. Modalities of pelvic autonomic nerve-sparing surgery in pelvic malignancies. [Article in Chinese]. China Oncol. 2006; 16:907-10.

16. Zang RY, Chen X, Tang J, Yang HJ. Preliminary study of pelvic autonomic nerve-sparing sub-radical/radical hysterectomy on preserving postsurgical bladder function in patients with cervix and endometrioid cancer. [Article in Chinese]. Prog Obstet Gynecol. 2006; 15:773-5. 
17. Fujii S, Takakura K, Matsumura N, Higuchi T, Yura S, Mandai M, Baba T, Yoshioka S. Anatomic identification and functional outcomes of the nerve sparing Okabayashi radical hysterectomy. Gynecol Oncol. 2007; 107:4-13.

18. Fujii S. Anatomic identification of nerve-sparing radical hysterectomy: a step-by-step procedure. Gynecol Oncol. 2008; 111:S33-41.

19. Querleu D, Morrow CP. Classification of radical hysterectomy. Lancet Oncol. 2008; 9:297-303.

20. Charoenkwan K. A simplified technique for nerve-sparing type III radical hysterectomy: by reorganizing their surgical sequence, surgeons could more easily identify key nerves. Am J Obstet Gynecol. 2010; 203:1-6.

21. Obesity: preventing and managing the global epidemic. Report of a WHO consultation. World Health Organ Techn Rep Ser. 2000; 894:i-xii, 1-253.

22. Liang Z, Chen Y, Xu H, Li Y, Wang D. Laparoscopic nervesparing radical hysterectomy with fascia space dissection technique for cervical cancer: description of technique and outcomes. Gynecol Oncol. 2010; 119:202-7.
23. Kavallaris A, Hornemann A, Chalvatzas N, Luedders D, Diedrich K, Bohlmann MK. Laparoscopic nerve-sparing radical hysterectomy: description of the technique and patients' outcome. Gynecol Oncol. 2010; 119:198-201.

24. Raspagliesi F, Ditto A, Fontanelli R, Solima E, Hanozet F, Zanaboni F, Kusamura S. Nerve-sparing radical hysterectomy: a surgical technique for preserving the autonomic hypogastric nerve. Gynecol Oncol. 2004; 93:307-14.

25. Kato K, Suzuka K, Osaki T, Tanaka N. Unilateral or bilateral nerve-sparing radical hysterectomy: a surgical technique to preserve the pelvic autonomic nerves while increasing radicality. Int J Gynecol Cancer. 2007; 17:1172-8.

26. Charoenkwan K, Srisomboon J, Suprasert P, Tantipalakorn C, Kietpeerakool C. Nerve-sparing class III radical hysterectomy: a modified technique to spare the pelvic autonomic nerves without compromising radicality. Int $\mathrm{J}$ Gynecol Cancer. 2006; 16:1705-12. 